Contents List available at VOLKSON PRESS Intelligent Computing and Information Engineering (ICIE ) DOI : http://doi.org/10.26480/icie.01.2017.45.47 Journal Homepage: : https://www.intelcomp-design.com/

\title{
DESIGN OF JSP-BASED FLASH SALE SYSTEM FOR VEGETABLE AND FRUIT MARKET
}

Tan Qiuhong*, Long Qi, Wang Qianjin

Yunnan Normal University, Kunming, China

*Corresponding author email: tanqiuhong1@126.com

This is an open access article distributed under the Creative Commons Attribution License, which permits unrestricted use, distribution, and reproduction in any medium, provided the original work is properly cited

\section{ARTICLE DETAILS}

\section{Article History:}

\section{Received 12 May2017}

Accepted 12 July 2017

Available online 14 September 2017

Keywords:

flash sale, JSP, Servlet, MVC, MySQL.

\section{ABSTRACT}

With the rapid development of information technology and the improvement of modern network technology, ecommerce is more and more mature, developing into a new business model. For example: Amazon, Alibaba, in addition to some brand flash sale sites: Vipshop, JUMEI, etc. Nevertheless, most of these sites are integrated largescale sites, involving various types of goods while lacking pertinence. In this paper, the studied shopping sites are constructed for system of flash sale in fresh fruits and vegetables market. This paper will design information system based on JSP website development and design language for flash sale in fruit and vegetable market, and save the data in MySQL database, so that consumers can buy satisfactory, assured and safe fresh fruits and vegetables conveniently, fast and efficiently.

\section{Introduction}

In recent years, the Internet is rapidly developing, reaching clothing, food, shelter, travel aspects in daily life. Especially in recent years, online shopping of fresh fruits and vegetables, seafood, etc. has been favored by a lot of customers. However, current domestic online shopping of fresh fruits and vegetables has not yet covered all the country, only active in economically developed areas with convenient transportation [1]. Fresh fruits and vegetables feature timeliness, after a certain period of time, fresh taste of the delivered fruits and vegetables cannot be accurately guaranteed, which is one hindrance to rapid development of online flash sale of fresh fruits and vegetables. In daily life, the domestic large and small farmer markets always provide fresh and delicious fruits and vegetables for the residents of the nearby areas, but oversupply is often the case, making many fresh fruits and vegetables slowly rot [2]. Meanwhile, office workers and lazy people cannot make time to pick their favorite fresh fruits and vegetables. Therefore, considering the above several problems in real life, this paper proposes a flash sale system for fruit and vegetable market in local areas.

\section{USED TECHNOLOGY FOR THE SYSTEM}

\subsection{MyEclipse}

\subsubsection{MyEclipse development environment}

MyEclipse is very powerful, and can support a wide range of open source products, so that developers will not suffer technology limitations during product development but make continuous innovations. Thanks to MyEclipse, simultaneous development is possible, so that developers can have access to complete technology provision during development with MyEclipse.

\subsection{MySQL 2.2 MySQL database}

MySQL is an associated database type management system, which has been widely welcomed among a number of small and medium-sized databases. MySQL is mostly characterized by fast speed, small volume. With a not high total cost, it is also an open source code. For Web application, it can be said to be excellent software for relationship type database management system.

\subsection{JSP and Servlet}

JSP is essentially a special JAVA language. JSP language has many advantages. First, JSP language can run directly despite editing location; second, JSP can work in many platform systems, that is, JSP is able to deploy the system in any environment, and can expand the task environment; third, JSP language enjoys diversified development tools with strong scalability [3]. As a result, JSP dynamic web technology is more appropriate for the design in this paper.

Servlet is a JAVA language-edited Web application server that can generate dynamic web pages. In most cases, Servlet is only used to extend HTTP protocolbased Web server. When a Web browser or other HTTP client programs issue a corresponding request, Servlet acts as a carrier between the database and the application.

\subsection{Tomcat server}

Tomcat Server, a free and open source Servlet container and web application server, belongs to a lightweight application server. So far, many small and medium-sized systems have been widely used in access through Tomcat server. Meanwhile, Tomcat server is also the first choice condition for development, debugging of JSP web technology.

\subsection{MVC programming mode}


MVC model is mainly composed of three parts: model, view and controller. The advantage of MVC design pattern is that you can have multiple views correspond to the same model. In the face of the current rapid technology development and needs of users at each level, MVC design pattern is gradually developing into an application available in a variety of ways.

\section{REALIZATION OF SYSTEM FUNCTIONS}

\subsection{The design of user registration and login module}

To enter the system for flash sale, consumers must first register or log on to the existing account. Users can directly click "buy immediately" on the product or click "registration", "login" button in the menu bar for this purpose. In the registration page of fruits and vegetables sale, the registration information mainly comprises six parts of user name, password, confirmation password, e-mail, nickname and contact telephone. The user must fill in the information according to the standard format for registration. After submission, the system will automatically judge correctness of the filled information [4]. There will be corresponding hint for wrongly filled information, so that users register successfully. The user's privacy and shopping security will be guaranteed. After successful registration, the page will directly jump to login page. The user should fill out the correct user name, password and verification code in the login page before successful login; otherwise there will be a corresponding hint. The page will directly jump to shopping page after successful login.

\subsection{Design of commodity query module}

After entry into the flash sale home page, the user administrator can directly search the product name in the current flash sale. The search bar hints input of "fruit, vegetables" in gray font. After the user enters key words of relevant flash sale items and clicks the "search" button or directly presses the Enter key, the system will quickly verify and process the query information. If there exist vegetables and fruits for the current flash sale, the page will automatically pull down to the column for flash sale items. Otherwise, a dialog box will pop up, suggesting that there are no queryable goods, and the previous wrong search information will be automatically deleted. Through this query mode, users can directly enter related key words of fruits and vegetables for flash sale, so that users can quickly and easily find wanted fruits and vegetables and experience the fun of "flash sale".

\subsection{Design for flash sale of fruits and vegetables}

One of the biggest features of the fruit and vegetable shopping system designed in this paper is flash sale. The flash sale deadline minus the current time shows the consumers the remaining time for the flash sale, and the flash sale information is count down in sequence in time units of day, hour, minute, second. Within the valid time of flash sale, the timer will display the remaining time, meanwhile "flash sale" button of each product is in bright color, indicating that flash sale is available. Click on the button and the page will jump to the shopping list page. When valid time of the flash sale is gone, the timer hints end of the current flash sale. Meanwhile, the button is in gray color, indicating end of the current flash sale and unavailability of any shopping.

\subsection{Design for user shopping cart management}

During the prescribed period for flash sale, click "buy immediately" in the flash sale page, the page will jump to the shopping cart page. The shopping cart includes information about pictures, product names, unit prices, quantities and subtotal of the purchased fruits and vegetables in flash sale as well as functions of single operation, all deletion and submission. The default value of product quantity is 1 , the user can arbitrarily add or subtract the quantity in the text box, and the subtotal price will be accordingly added or subtracted [5]. Users can also click "delete" in the operation to delete any already selected fruits and vegetables. If the user wants to empty all the fruits and vegetables in the shopping cart, click the "delete" button on the right bottom, and the shopping cart page will automatically shut down. If the user clicks multiple products, multiple shopping cart pages will open, and the original page will not be refreshed. But if the user clicks the same fruits and vegetables several times, the quantity in the shopping cart will automatically increase, without repeated information shown. Finally, in the "My Shopping Cart" column, the user must select the delivery address; otherwise, the shopping information will not be submitted. After all the information is confirmed to be submitted, the shopping cart page will automatically shut down and the submitted data will be sent to the database.

\section{DESIGN OF SYSTEM DATABASE MANAGEMENT}

We need to analyze and record the collected system data through the database, so as to facilitate the processing of the data obtained in the system design. According to the characteristics of flash sale of fruits and vegetables market, the data tables needed for the system are as follows:

Table1: Registered User Basic Information Table

\begin{tabular}{|l|l|l|l|}
\hline Fieldname & Field type & Column name & Explanation \\
\hline Username & Varchar & id & Major key \\
\hline Psw & Varchar & User login password & \\
\hline Email & Varchar & E-mail & \\
\hline Nickname & Varchar & Nickname & \\
\hline Phone & Varchar & User contact number & \\
\hline
\end{tabular}

Table2: Query Fruits and Vegetables Information Table

\begin{tabular}{|l|l|l|l|}
\hline Fieldname & Field type & Column name & Explanation \\
\hline Id & Int & id & Major key \\
\hline Allname & Varchar & Origin of fruits and vegetables & \\
\hline Proname & Varchar & Information of fruits and vegetables & \\
\hline Imgurl & Varchar & Picture of fruits and vegetables & \\
\hline Price & Varchar & Price of fruits and vegetables & \\
\hline
\end{tabular}

Table 3: User Shopping Cart Table

\begin{tabular}{|l|l|l|l|}
\hline Fieldname & Field type & Columunname & Explanation \\
\hline Id & Int & id & Major key \\
\hline Username & Varchar & User Logon Name & \\
\hline Allname & Varchar & Origin of fruits and vegetables & \\
\hline Proname & Varchar & Information of fruits and vegetables & \\
\hline Imgurl & Varchar & Picture of fruits and vegetables & \\
\hline Price & Varchar & Total price of fruits and vegetables & \\
\hline Num & Int & Total quantity of fruits and vegetables & \\
\hline
\end{tabular}




\begin{tabular}{|l|l|l|l|}
\hline Phone & Varchar & User contact number & \\
\hline Address & Varchar & User delivery area & \\
\hline
\end{tabular}

Table4: User Delivery Area Selection Table

\begin{tabular}{|l|l|l|l|}
\hline Fieldname & Field type & Columunname & Explanation \\
\hline Id & Int & id & Major key \\
\hline Zone & Varchar & Region type & \\
\hline Type & Varchar & Regional initial type & \\
\hline Subtype & Varchar & Regional alphabet sort selection & \\
\hline
\end{tabular}

\section{CONCLUSIONS}

Through feasibility analysis of flash sale system in fruit and vegetable market of regional extent and design of functional module management, the flash sale system based on Java-based JSP development language, MVC model and MySQL database designed in this paper will provide more conveniences for the office workers, lazy people and a larger part of groups, reducing unnecessary waste of space and time. Moreover, it effectively and conveniently solves problems of stockpile, poor efficiency and manpower waste, etc. of sellers of fruits and vegetables market, which can quickly promote economic development of physical regional fruit and vegetable market to a certain extent.

\section{REFERENCES}

[1] Lifei, C. 2012. JSP-based online shopping system development. Suzhou University.

[2] Zongfei, H. Design and Implementation of JSP- Based Online Meal Booking System. University of Electronic Science and Technology of China.

[3] Zhaoming, L. 2013. Commercial (shopping) website design and making. University of Electronic Science and Technology of China.

[4] Zhaobin, H. 2012. Online bookstore system design and implementation based on JSP technology. University of Electronic Science and Technology of China.

[5] Yuandong, L. 2010. Design and Implementation of JSP Technology-Based E-commerce Website. South China University of Technology. 